Bull. Austral. Math. Soc.

VoL. 55 (1997) [453-467]

\title{
NILPOTENTS IN SEMIGROUPS OF PARTIAL TRANSFORMATIONS
}

\begin{abstract}
R.P. Sullivan
In 1987, Sullivan determined when a partial transformation $\alpha$ of an infinite set $X$ can be written as a product of nilpotent transformations of the same set: he showed that when this is possible and the cardinal of $X$ is regular then $\alpha$ is a product of 3 or fewer nilpotents with index at most 3 . Here, we show that 3 is best possible on both counts, consider the corresponding question when the cardinal of $X$ is singular, and investigate the role of nilpotents with index 2 . We also prove that the nilpotent-generated semigroup is idempotent-generated but not conversely.
\end{abstract}

\section{INTRODUCTION}

Throughout this paper $X$ will denote an infinite set with cardinal $m$, and if $n$ is any cardinal then $n^{\prime}$ will denote the successor of $n$ (that is, the least cardinal greater than $n$ ). All notation and terminology will be from [1] and [3] unless specified otherwise. In particular, $\mathcal{T}(X)$ denotes the full transformation semigroup on $X$. If $\alpha \in \mathcal{T}(X)$, we let $r(\alpha)$ denote the rank of $\alpha$ (that is, $|X \alpha|$ ) and put

$$
\begin{array}{llrl}
D(\alpha) & =X \backslash X \alpha, & & d(\alpha)=|D(\alpha)|, \\
S(\alpha) & =\{x \in X: x \alpha \neq x\}, & s(\alpha)=|S(\alpha)|, \\
C(\alpha)=\bigcup\left\{y \alpha^{-1}:\left|y \alpha^{-1}\right| \geqslant 2\right\}, & c(\alpha)=|C(\alpha)| .
\end{array}
$$

The cardinal numbers $d(\alpha), s(\alpha)$ and $c(\alpha)$ are called, respectively, the defect, the shift and the collapse of $\alpha$ and were originally used by Howie [2] to characterise the elements of $\mathcal{T}(X)$ that can be written as a product of idempotents in $\mathcal{T}(X)$. In particular, he later showed [4] that the set

$$
Q_{m}=\{\alpha \in \mathcal{T}(X): d(\alpha)=s(\alpha)=c(\alpha)=m\}
$$

is an idempotent-generated subsemigroup of $\mathcal{T}(X)$. Later still, in [6] Marques considered the Rees quotient semigroup $P_{m}=Q_{m} / I_{m}$ where $I_{m}=\left\{\alpha \in Q_{m}: r(\alpha)<m\right\}$, an ideal of $Q_{m}$.

\section{Received 24 June 1996}

This paper was completed while visiting Dr Peter Trotter at the University of Tasmania. I thank him and his colleagues for their generous hospitality during my stay.

Copyright Clearance Centre, Inc. Serial-fee code: 0004-9729/97 \$A2.00+0.00. 
Among other things, she proved that for any infinite $m$, every element of $P_{m}$ is a product of 4 or fewer idempotents and that 4 is best possible. Then in [5] the authors showed that if $m$ is a regular cardinal, the set

$$
K_{m}=\left\{\alpha \in P_{m}:\left|y \alpha^{-1}\right|=m \text { for some } y \in X\right\} \cup\{0\}
$$

equals the subsemigroup of $P_{m}$ generated by the nilpotents in $P_{m}$. And in [7] the authors proved that if $m$ is singular (that is, non-regular) then the subsemigroup of $P_{m}$ generated by its nilpotents equals the set

$$
L_{m}=\left\{\alpha \in P_{m}: \text { for each } p<m \text {, there exists } y \in X \text { such that }\left|y \alpha^{-1}\right|>p\right\} \cup\{0\} .
$$

Moreover, from [5] and [7] we know that each element of $K_{m}$ and of $L_{m}$ is a product of 3 or fewer nilpotents with index 2 (that is, $\lambda \neq 0$ and $\lambda^{2}=0$ ) and that 3 is best possible.

Let $\mathcal{P}(X)$ denote the semigroup of all partial transformations of $X$ and if $\alpha \in$ $\mathcal{P}(X)$, write $g(\alpha)=|X \backslash \operatorname{dom} \alpha|$ and call this the gap in $\alpha$. In [9, Corollary 3], I proved that if $m$ is regular then the set

$$
\begin{aligned}
\mathcal{L}(X)=\{\alpha \in \mathcal{P}(X): d(\alpha)=m, g(\alpha) & \geqslant 1, \\
& \text { and } \left.\left|y \alpha^{-1} \cup(X \backslash \operatorname{dom} \alpha)\right|=m \text { for some } y \in X\right\}
\end{aligned}
$$

is the subsemigroup of $\mathcal{P}(X)$ generated by the nilpotents in $\mathcal{P}(X)$. Moreover, in this case, $\mathcal{L}(X)$ is regular and each of its elements equals a product of 3 or fewer nilpotents with index at most 3 . In this paper, we show that $\mathcal{L}(X)$ is idempotent-generated; and provide bounds on the number of nilpotents (and their indices) required to express each element of $\mathcal{L}(X)$ as a product of nilpotents: we show, for example, that both the product 3 and the index 3 just mentioned are best possible. We also investigate analogous questions when $m$ is singular.

\section{NiLPOTENTS AS GENERATORS: THE REgULAR CARDINAL CASE}

We extend the convention introduced in [1, vol.2, p.241]: namely, if $\alpha \in \mathcal{P}(X)$ is non-zero then we write

$$
\alpha=\left(\begin{array}{c}
A_{i} \\
x_{i}
\end{array}\right)
$$

and take as understood that the subscript $i$ belongs to some (unmentioned) index set $I$, that the abbreviation $\left\{x_{i}\right\}$ denotes $\left\{x_{i}: i \in I\right\}$, and that $X \alpha=$ ran $\alpha=\left\{x_{i}\right\}$, $A_{i}=x_{i} \alpha^{-1}$ and $\operatorname{dom} \alpha=\bigcup A_{i}$.

To compare the results in [9, Section 3] with those in [5] and [7], we let $\phi \notin X$, put $X^{\phi}=X \cup \phi$ and

$$
F_{\phi}=\left\{\alpha \in \mathcal{T}\left(X^{\phi}\right): \phi \alpha=\phi\right\}
$$


and define $\theta: \mathcal{P}(X) \rightarrow F_{\phi}, \alpha \rightarrow \alpha \theta$, where $x(\alpha \theta)=x \alpha$ if $x \in \operatorname{dom} \alpha$ and $x(\alpha \theta)=\phi$ otherwise. Clearly, $\theta$ is an isomorphism and, when $m$ is regular, the image of $\mathcal{L}(X)$ under $\theta$ is the semigroup

$$
L_{\phi}=\left\{\alpha \in F_{\phi}: d(\alpha)=m,\left|\phi \alpha^{-1}\right| \geqslant 2 \text {, and }\left|y \alpha^{-1}\right|=m \text { for some } y \in X^{\phi}\right\} .
$$

Note that $L_{\phi}$ contains the ideal $I_{\phi}=\left\{\alpha \in L_{\phi}: r(\alpha)<m\right\}$ and the Rees quotient semigroup $L_{\phi} / I_{\phi}$ can be identified with $\left\{\alpha \in L_{\phi}: r(\alpha)=m\right\} \cup 0$, where 0 represents the zero of $L_{\phi} / I_{\phi}$. In this way, $L_{\phi} / I_{\phi}$ can be regarded as the semigroup

$$
K_{m}(\phi)=\left\{\alpha \in K_{m}\left(X^{\phi}\right): \phi \alpha=\phi \text { and }\left|\phi \alpha^{-1}\right| \geqslant 2\right\} \cup 0 .
$$

In [5] the authors showed that every non-zero $\alpha \in K_{m}\left(X^{\phi}\right)$ equals a product of nilpotents in $K_{m}\left(X^{\phi}\right)$ with index 2. This is also true of $K_{m}(\phi)$. For, if $\alpha, \beta \in$ $K_{m}\left(X^{\phi}\right)$ and $\phi \alpha \beta=\phi$ then there exist $\alpha^{\prime} \in K_{m}\left(X^{\phi}\right)$ and $\beta^{\prime} \in K_{m}(\phi)$ such that $\alpha \beta=\alpha^{\prime} \beta^{\prime}, \operatorname{ker} \alpha=\operatorname{ker} \alpha^{\prime}$ and $\phi \alpha^{\prime}=\phi$. To see this, suppose $\phi \alpha=a, \phi \beta=b$ and consider two cases. If $\phi \notin X \alpha$, we let $x \alpha^{\prime}=\phi$ for $x \in a \alpha^{-1}$ and $x \alpha^{\prime}=x \alpha$ otherwise, and let $x \beta^{\prime}=\phi$ for $x \in \phi \beta^{-1} \cup \phi$ and $x \beta^{\prime}=x \beta$ otherwise. On the other hand, if $\phi \in X \alpha \backslash a$, we choose $d \notin X \alpha$ and let $x \alpha^{\prime}=\phi$ for $x \in a \alpha^{-1}, x \alpha^{\prime}=a$ for $x \in \phi \alpha^{-1}$ and $x \alpha^{\prime}=x \alpha$ otherwise, and let $x \beta^{\prime}=\phi$ for $x \in\left(\phi \beta^{-1} \backslash a\right) \cup \phi \cup d, x \beta^{\prime}=b$ for $x \in\left(b \beta^{-1} \backslash \phi\right) \cup a$ and $x \beta^{\prime}=x \beta$ otherwise. It is now easy to check that, in both cases, $\alpha^{\prime}$ and $\beta^{\prime}$ possess the required properties. Moreover, since there is little difference between the ranges of $\alpha$ and $\alpha^{\prime}$ (and the kernels of $\beta$ and $\beta^{\prime}$ ) it is clear that $\alpha^{\prime}$ is nilpotent in $K_{m}\left(X^{\phi}\right)$ if and only if $\alpha$ is (likewise for $\beta$ and $\beta^{\prime}$ ). Consequently, if $\alpha \in K_{m}(\phi)$ then $\alpha$ is a product of nilpotents with index 2 in $K_{m}\left(X^{\phi}\right)$ and, by the foregoing remark, these can be assumed to lie in $K_{m}(\phi)$.

Having said all this, it will transpire from what follows that $L_{\phi}$, the inverse image of $K_{m}(\phi)$ under the natural map $L_{\phi} \rightarrow L_{\phi} / I_{\phi}$, is not generated by its nilpotents with index 2 (that is, $\lambda \in L_{\phi}$ such that $\lambda^{2}=\phi$ but $\lambda \neq \phi$ where, in this context, $\phi$ denotes the constant transformation in $\mathrm{L}_{\phi}$ ). In particular, it will be clear that if $X=\left\{a_{i}\right\} \cup\left\{b_{i}\right\} \cup x$ then

$$
\lambda=\left(\begin{array}{ccc}
a_{i} & \left\{b_{i}\right\} & \{x, \phi\} \\
b_{i} & x & \phi
\end{array}\right)
$$

cannot be written as a product of nilpotents in $L_{\phi}$ with index 2 but, as already shown, as an element of $K_{m}(\phi)$ it does equal a product of nilpotents in $K_{m}(\phi)$ with index 2 . In addition, whereas $K_{m}(\phi)$ is 0-bisimple (compare [7, Theorem 2.1]) the same is not true of $L_{\phi}$. That is, very little information about $L_{\phi}$ can be obtained directly from [5] and [7], so we continue to work within $\mathcal{L}(X)$ itself.

The cofinality of $m, \operatorname{cf}(m)$, plays a fundamental role in what follows: since it is difficult to find an elementary account of the relevant facts in the literature, we summarise them in the following way, using [10, Theorem A.3.9] as our authority. 
THEOREM 2.1. Suppose $m$ is an arbitrary infinite cardinal. Then $\mathrm{cf}(m)$ is the least cardinal $n$ such that $m$ can be expressed as a sum of $n$ cardinals each less than $m$. Hence, $\operatorname{cf}(m) \leqslant m$ where equality occurs if and only if $m$ is regular. In particular, both $\mathrm{cf}(m)$ and $m^{\prime}$ are regular cardinals. If $m$ is singular then $\mathrm{cf}(m)$ is infinite and $m$ can be expressed as the sum of a strictly increasing sequence of $\mathrm{cf}(m)$ cardinals each less than $m$.

For convenience, we recall the following result from [9, Theorem 3, p.336 and p.341].

THEOREM 2.2. If $m$ is a regular cardinal then the semigroup $\mathcal{L}(X)$ is regular and each $\alpha \in \mathcal{L}(X)$ equals a product of 3 or fewer nilpotents with index at most 3 . Moreover, $\mathcal{L}(X)$ contains the ideal $I_{m}^{*}=\{\alpha \in \mathcal{P}(X): r(\alpha)<m\}$.

The proof of Theorem 2.2 involves two cases: namely whether $g(\alpha)=m$ or $g(\alpha)<$ $m$, and in the first case $\alpha$ can be written as a product of 3 or fewer nilpotents with index 2 . We begin by characterising precisely when $\alpha$ is a product of nilpotents with index 2: besides its intrinsic interest, the next result shows that the index 3 in Theorem 2.2 is best possible.

THEOREM 2.3. Suppose $m$ is an arbitrary infinite cardinal and $\alpha \in \mathcal{P}(X)$ is non-zero. Then $\alpha$ is a product of nilpotents with index 2 if and only if $d(\alpha)=m$ and $g(\alpha) \geqslant r(\alpha)$. Moreover, when this occurs, $\alpha$ is a product of 3 or fewer nilpotents with index 2 .

Proof: If $\lambda^{2}=\emptyset$ then $X \lambda \subseteq X \backslash$ dom $\lambda$ : that is, $r(\lambda) \leqslant g(\lambda)$ and, by [9, Lemmas 11 and 13] (compare Lemma 3.2 below), $d(\lambda)=m$. Hence, any nilpotent with index 2 satisfies the given conditions. Consequently, if $\lambda_{1} \ldots \lambda_{r}$ is a product of such nilpotents then $d\left(\lambda_{1} \ldots \lambda_{r}\right)=m$ and

$$
r\left(\lambda_{1} \ldots \lambda_{r}\right) \leqslant r\left(\lambda_{1}\right) \leqslant g\left(\lambda_{1}\right) \leqslant g\left(\lambda_{1} \ldots \lambda_{r}\right)
$$

since $d(\beta) \leqslant d(\alpha \beta)$ and $r(\alpha \beta) \leqslant \min (r(\alpha), r(\beta))$ for all $\alpha, \beta \in \mathcal{P}(X)$.

Conversely, suppose $\alpha$ satisfies the given conditions: what follows is essentially the argument in the first paragraph of the proof of [9, Theorem 3]. Suppose $X \alpha=\left\{x_{i}\right\}$ and $A_{i}=x_{i} \alpha^{-1}$. If $|(X \backslash \operatorname{ran} \alpha) \cap(X \backslash \operatorname{dom} \alpha)| \geqslant r(\alpha)$ then we can choose $c_{i} \in$ $(X \backslash \operatorname{ran} \alpha) \cap(X \backslash \operatorname{dom} \alpha)$ and write

$$
\alpha=\left(\begin{array}{l}
A_{i} \\
c_{i}
\end{array}\right) \circ\left(\begin{array}{l}
c_{i} \\
x_{i}
\end{array}\right)
$$

where each transformation on the right is nilpotent with index 2. Suppose instead that $|(X \backslash \operatorname{ran} \alpha) \cap(X \backslash \operatorname{dom} \alpha)|<r(\alpha)$. In this event, if $r(\alpha)$ is finite, we choose 
$c_{i} \in X \backslash \operatorname{dom} \alpha$ and $d_{i} \in(X \backslash X \alpha) \backslash\left\{c_{i}\right\}$, and put

$$
\alpha=\left(\begin{array}{l}
A_{i} \\
c_{i}
\end{array}\right) \circ\left(\begin{array}{l}
c_{i} \\
d_{i}
\end{array}\right) \circ\left(\begin{array}{l}
d_{i} \\
x_{i}
\end{array}\right)
$$

where each transformation on the right is again nilpotent with index 2 . On the other hand, if $r(\alpha)$ is infinite then $|X \alpha \cap(X \backslash \operatorname{dom} \alpha)|=r(\alpha)$, so we can choose $c_{i} \in X \alpha \cap$ $(X \backslash \operatorname{dom} \alpha)$ and $d_{i} \in X \backslash X \alpha$ to ensure that the above decomposition of $\alpha$ remains valid.

To show that 3 is best possible in the above result, we need to characterise when $\alpha$ is a product of 2 nilpotents with index 2 , and for this we need to describe Green's relations on $\mathcal{L}(X)$. The following characterisation of Green's relations on $\mathcal{P}(X)$ is well-known: its proof is entirely similar to that given in [1, vol. 1, pp.52-53] for $\mathcal{T}(X)$, and so is omitted.

Lemma 2.4. If $\alpha, \beta \in \mathcal{P}(X)$ then

(a) $\alpha \mathcal{L} \beta$ if and only if $X \alpha=X \beta$,

(b) $\alpha \mathcal{R} \beta$ if and only if $\operatorname{ker} \alpha=\operatorname{ker} \beta$,

(c) $\alpha \mathcal{D} \beta$ if and only if $r(\alpha)=r(\beta)$, and

(d) $\mathcal{D}=\mathcal{J}$

The regularity of $\mathcal{L}(X)$ when $m$ is regular was established in [9, p.341]. For what follows, we need a more general result.

Lemma 2.5. If $m$ is an arbitrary infinite cardinal then $\mathcal{L}(X)$ is a regular semigroup.

Proof: We suppose $m$ is singular and let $\alpha \in \mathcal{L}(X)$. Write $X \alpha=\left\{x_{i}\right\}$ and $A_{i}=x_{i} \alpha^{-1}$. Choose $a_{i} \in A_{i}$ and define a transformation $\beta$ by letting $\operatorname{dom} \beta=\left\{a_{i}\right\}$ and $a_{i} \beta=x_{i}$ for each $i \in I$. Then $g(\beta)=m$ since $d(\alpha)=m$, and $d(\beta)=m$ whenever $r(\alpha)<m$. If $r(\alpha)=m$ then, by [9, Theorem 4], $g(\alpha)=m$ or $\alpha$ is spread over $m$. In the former case, $d(\beta)=m$; and in the latter case, we know $\left|\bigcup A_{p}\right|=m$ for some $P \subseteq I$ with $|P|=\operatorname{cf}(m)$ : that is, $X \backslash X \beta$ contains $\bigcup\left(A_{p} \backslash a_{p}\right)$, a set with cardinal $m$. Hence, by [9, Corollary 4], $\beta$ is a product of nilpotents and clearly, $\alpha=\alpha \beta \alpha$.

Since $\mathcal{L}(X)$ is a regular subsemigroup of $\mathcal{P}(X)$, it follows from [3, Proposition II.4.5] that the $\mathcal{L}$ and $\mathcal{R}$ relations on $\mathcal{L}(X)$ can be described just as in Lemma 2.4. The reason for noting this fact will be apparent after we quote the following result from [5, Lemma 2.5].

Lemma 2.6. Let $T$ be a regular semigroup with a zero 0 . If $a \in T$ and $a=x y$ for some nilpotents $x, y$ in $T$ with index 2 then $a=x_{1} y_{1}$ for some nilpotents $x_{1}, y_{1}$ in $T$ with index 2 such that $x_{1} \mathcal{R} a$ and $y_{1} \mathcal{L} a$.

The next result should be compared with [5, Proposition 2.4] and [7, Lemma 2.2]. 
THEOREM 2.7. Suppose $m$ is an arbitrary infinite cardinal and $\alpha \in \mathcal{P}(X)$ is non-zero. Then $\alpha$ is a product of 2 nilpotents with index 2 if and only if $|(X \backslash \operatorname{ran} \alpha) \cap(X \backslash \operatorname{dom} \alpha)| \geqslant r(\alpha)$.

Proof: The second paragraph in the proof of Theorem 2.3 shows that if the condition holds then $\alpha$ can be written as a product of two nilpotents with index 2 . So, we suppose $\alpha=\lambda \mu \neq \emptyset$ where $\lambda^{2}=\mu^{2}=\emptyset$. By Lemma 2.6, we can also assume $\operatorname{ker} \lambda=\operatorname{ker} \alpha$ and $X \mu=X \alpha$. Let $X \alpha=\left\{x_{i}\right\}, A_{i}=x_{i} \alpha^{-1}$ and $A_{i} \lambda=y_{i}$. Since $\lambda^{2}=\emptyset$, we know $\left\{y_{i}\right\} \subseteq X \backslash \operatorname{dom} \lambda=X \backslash \operatorname{dom} \alpha$. Suppose, for contradiction, that $|(X \backslash \operatorname{ran} \alpha) \cap(X \backslash \operatorname{dom} \alpha)|<r(\alpha)$. Then $\left\{y_{i}\right\} \cap X \alpha \neq \emptyset$ where each $y_{i} \in \operatorname{dom} \mu$. Hence, we have $X \mu^{2}=(X \alpha) \mu \supseteq\left(\left\{y_{i}\right\} \cap X \alpha\right) \mu \neq \emptyset$, contradicting $\mu^{2}=\emptyset$.

It remains to note that there exist $\alpha \in \mathcal{P}(X)$ with $d(\alpha)=m$ and $g(\alpha) \geqslant r(\alpha)$ but $|(X \backslash \operatorname{ran} \alpha) \cap(X \backslash \operatorname{dom} \alpha)|<r(\alpha)$. To see this, write $X=\left\{x_{i}\right\} \cup\left\{a_{i}\right\} \cup\left\{b_{i}\right\} \cup\left\{c_{j}\right\}$ where $|I|=m>|J|$ and put

$$
\alpha=\left(\begin{array}{cc}
x_{i} & a_{i} \\
x_{i} & b_{i}
\end{array}\right) .
$$

Note also that there are $\alpha \in \mathcal{P}(X)$ which cannot be written as a product of nilpotents with index 2. For example, if $X=\left\{a_{i}\right\} \cup\left\{b_{i}\right\} \cup x$ then

$$
\alpha=\left(\begin{array}{cc}
a_{i} & \left\{b_{i}\right\} \\
b_{i} & x
\end{array}\right)
$$

is a nilpotent with index 3 which does not satisfy the conditions of Theorem 2.3 (the need to consider such nilpotents did not arise in [5] and [7]).

The second case in the proof of Theorem 2.2 leads to $\alpha$ being written as a product of 3 of fewer nilpotents, the first of which has index 3 and the other two have index 2. We now show this occurs whenever $\alpha$ does not belong to $\mathcal{K}(X)$, the subsemigroup of $\mathcal{L}(X)$ generated by the nilpotents in $\mathcal{L}(X)$ with index 2 .

THEOREM 2.8. If $m$ is regular and $\alpha \notin \mathcal{K}(X)$ then $\alpha$ is the product of 3 or fewer nilpotents, the first of which has index 3 and lies outside $\mathcal{K}(X)$ and the other two have index 2.

Proof: If $\alpha \notin \mathcal{K}(X)$ then $g(\alpha)<r(\alpha)$ and so $g(\alpha)<m$. Hence, by [9, Corollary $3]$, some $z \alpha^{-1}$ has cardinal $m$. Then the second paragraph in the proof of $[9$, Theorem 3 ], shows that $\alpha$ is a product of 3 nilpotents, the first having index 3 and the other two having index 2 , and clearly the first cannot belong to $\mathcal{K}(X)$.

It is often possible to do better in the above result and write $\alpha \notin \mathcal{K}(X)$ as a product of just two nilpotents, the first having index 3 and the second having index at most 3 . The next result characterises when this occurs and at the same time shows that the product 3 is best possible in Theorem 2.2 . 
THEOREM 2.9. Suppose $m$ is regular and $\alpha \notin \mathcal{K}(X)$. Then $\alpha$ is a product of two nilpotents with index at most 3 if and only if there exists $z \in X$ such that $\left|z \alpha^{-1} \cap(X \backslash X \alpha)\right| \geqslant r(\alpha)$ and $(X \backslash \operatorname{dom} \alpha) \backslash z$ is non-empty.

Proof: Suppose $\alpha=\lambda \mu$ where $\lambda$ and $\mu$ are nilpotent with index at most 3 . If $\lambda$ has index 2 then $g(\lambda) \geqslant r(\lambda)$ : an argument similar to that in the first paragraph in the proof of Theorem 2.3 then shows that $\alpha$ must satisfy the same inequality, in which case $\alpha \in \mathcal{K}(X)$. Hence, $\lambda$ must have index 3. Let $X \alpha=\left\{x_{i}\right\}$ and $A_{i}=x_{i} \alpha^{-1}$. If $r(\alpha)<m$ then $\left|(X \backslash X \alpha) \cap A_{i}\right|=m$ for some $i \in I$ since $m$ is regular and, by supposition, $|(X \backslash X \alpha) \cap(X \backslash \operatorname{dom} \alpha)|<m$ and $d(\alpha)=m$. Hence, we now assume $r(\alpha)=m$.

Let $B_{i}=x_{i} \mu^{-1}$ and note this is non-empty since $X \alpha \subseteq X \mu$. Let ker $\lambda=\left\{D_{j}\right\}$ where $|J|=m$ (since $r(\lambda) \geqslant r(\alpha)=m$ ). Then each $A_{i}$ is a union of some $D_{j}$ and $A_{i} \lambda \subseteq B_{i}$ for each $i \in I$. Fix some $b_{i} \in A_{i} \lambda$ and write $b_{i} \lambda^{-1}=D_{i}$. Note that $\left\{b_{i}\right\} \subseteq$ $\operatorname{dom} \mu$, and $\mu$ maps $\left\{b_{i}\right\}$ in a one-to-one fashion onto $\left\{x_{i}\right\}$. Now, since $\alpha \notin \mathcal{K}(X)$ and $X \backslash \operatorname{dom} \lambda \subseteq X \backslash \operatorname{dom} \alpha,\left|\left\{b_{i}\right\} \cap X \backslash \operatorname{dom} \lambda\right|<m$ and hence $\|\left(\left\{b_{i}\right\} \cap D_{j}\right) \mid=m$. If $\left\{b_{i}\right\} \cap D_{j} \neq \emptyset$ for $m$ of the $D_{j}$ then $\left|\left\{b_{i}\right\} \lambda\right|=m$ : that is, $r\left(\lambda^{2}\right)=m$, contradicting the fact that $X \lambda^{2} \subseteq X \backslash \operatorname{dom} \lambda$ which has cardinal less than $m$. Hence, if $K=\{j \in$ $\left.J:\left\{b_{i}\right\} \cap D_{j} \neq \emptyset\right\}$ then $|K|<m$. But then $\bigcup\left(\left\{b_{i}\right\} \cap D_{k}\right)$ has cardinal $m$ and so $\left|\left\{b_{i}\right\} \cap D_{0}\right|=m$ for some index $0 \in K$ (since $m$ is regular). Note that $D_{0}$ is contained in some $A_{i}$ since $g(\alpha)<m$. Write $\left\{b_{i}\right\} \cap D_{0}=\left\{b_{p}\right\}$ and suppose, for contradiction, that $\left|(X \backslash X \alpha) \cap A_{i}\right|<m$ for all $i \in I$. Then in particular, $\left|(X \backslash X \alpha) \cap\left\{b_{p}\right\}\right|<m$ and so $X \alpha \cap\left\{b_{p}\right\}=\left\{b_{p 1}\right\}$ say, has cardinal $m$. Note that $\left\{b_{p_{1}}\right\} \mu$ has cardinal $m$. Since $\left\{b_{p 1}\right\} \subseteq\left\{x_{i}\right\}$, by the choice of the $b_{i}$ we know there exist $c_{p} \in\left\{b_{i}\right\}$ such that $c_{p} \mu=b_{p 1}$. We now repeat the foregoing argument with $\left\{c_{p}\right\}$ replacing $\left\{b_{i}\right\}$. That is, $\left\{c_{p}\right\}$ must intersect less than $m$ of the $D_{j}$ and so there is an index $1 \in J$ such that $\left|\left\{c_{p}\right\} \cap D_{1}\right|=m$. Once again, note that $D_{1}$ is contained in some $A_{i}$ and if $\left\{c_{p}\right\} \cap D_{1}=$ $\left\{d_{p}\right\}$ then $X \alpha \cap\left\{d_{p}\right\}=\left\{b_{p 2}\right\}$ say, has cardinal $m$. Then $\left\{b_{p 2}\right\} \mu^{2}$ has cardinal $m$ and we need only repeat the argument one more time to reach a contradiction (since $\mu$ has index at most 3 ).

In the last two paragraphs we have shown that $\left|(X \backslash X \alpha) \cap A_{0}\right| \geqslant r(\alpha)$ for some index $0 \in I$. We now prove that we can assume $(X \backslash \operatorname{dom} \alpha) \backslash x_{0}$ is non-empty. For, suppose $X \backslash \operatorname{dom} \alpha=x_{0}$ (recall that $g(\alpha) \geqslant 1$ ). Then $\emptyset \neq(X \lambda) \lambda \subseteq X \backslash$ $\operatorname{dom} \lambda=x_{0}$ implies that $x_{0} \lambda^{-1}=Y$ say, contains $X \lambda$ and so its cardinal is at least $r(\alpha)$. In addition, since $g(\alpha)<r(\alpha), x_{0}$ must belong to $\operatorname{dom} \mu, x_{0} \mu=x_{1}$ say. If $|X \lambda \cap(X \backslash X \alpha)| \geqslant r(\alpha)$ then $\left|A_{1} \cap(X \backslash X \alpha)\right| \geqslant r(\alpha)$ since $A_{1}=x_{1} \alpha^{-1}$ contains $Y$. Since $x_{1} \neq x_{0}$ ( $\mu$ is nilpotent) and $X \backslash \operatorname{dom} \alpha=x_{0}$ by supposition, the set $A_{1}$ possesses the desired properties. Thus, we assume $|X \lambda \cap(X \backslash X \alpha)|<r(\alpha)$ and deduce that $X \lambda \cap X \alpha=\left\{x_{p}\right\}$ say, is non-empty (possibly $r(\alpha)$ is finite). But then 
there exist $e_{p} \in X \lambda \subseteq Y$ such that $e_{p} \mu=x_{p}$ and $\left|\left\{e_{p}\right\} \cap(X \backslash X \alpha)\right|<r(\alpha)$ implies $\left\{e_{p}\right\} \cap X \alpha=\left\{x_{q}\right\}$ say, is non-empty. Once again, there exist $e_{q} \in X \lambda$ such that $e_{q} \mu=x_{q}$ and now $\left\{e_{q}\right\} \mu^{2}=\left(\left\{e_{p}\right\} \cap X \alpha\right) \mu$ is non-empty (since each $e_{p} \in \operatorname{dom} \mu$ ). Clearly, this argument can be repeated once more to find that $\mu^{3} \neq \emptyset$, a contradiction.

Conversely, suppose $Y=z \alpha^{-1}$ and $|Y \cap(X \backslash X \alpha)| \geqslant r(\alpha)$. Let $X \alpha \backslash z=\left\{x_{i}\right\}$ and $A_{i}=x_{i} \alpha^{-1}$. Choose $b_{i} \in Y \cap(X \backslash X \alpha)$ and $c \in(X \backslash \operatorname{dom} \alpha) \backslash z$, and note that

$$
\alpha=\left(\begin{array}{cc}
A_{i} & Y \\
b_{i} & c
\end{array}\right) \circ\left(\begin{array}{ll}
b_{i} & c \\
x_{i} & z
\end{array}\right)
$$

where the first transformation on the right is nilpotent with index 3 and the second is nilpotent with index at most 3 (one $x_{i}$ may equal $c$ ).

It remains to note that there exist $\alpha \notin \mathcal{K}(X)$ which do not satisfy the conditions of Theorem 2.9: for example, the transformation defined in (1).

\section{Nilpotents as generators: the singular Cardinal CaSe}

Throughout this section $m$ will be a singular infinite cardinal. In this context, we say $\alpha \in \mathcal{P}(X)$ is spread over its rank if, for each $p<r(\alpha)$, some $z \alpha^{-1}$ has cardinal greater than $p$. In [7] the authors showed that, when $m$ is singular, the set

$$
L_{m}=\left\{\alpha \in P_{m}: \alpha \text { is spread over } m\right\} \cup\{0\}
$$

equals the subsemigroup of $P_{m}$ generated by the nilpotents of $P_{m}$. Moreover, each $\alpha \in L_{m}$ is a product of 3 or fewer nilpotents with index 2 in $P_{m}$, and 3 is best possible. This is comparable with the following result from [9, Theorem 4]: note that the proof in $[9, \mathrm{p} .340]$ involves a nilpotent $\lambda$ which is stated to have index 3 but in fact has index 4 .

Theorem 3.1. Suppose $m$ is singular and $\alpha \in \mathcal{P}(X)$. Then $\alpha \in \mathcal{L}(X)$ if and only if $g(\alpha) \geqslant 1, d(\alpha)=m$ and either $g(\alpha) \geqslant r(\alpha)$ or $\alpha$ is spread over its rank. Moreover, when this occurs, $\alpha$ can be written as a product of 4 or fewer nilpotents with index at most 4.

In Section 2, we characterised when $\alpha \in \mathcal{P}(X)$ is a product of nilpotents with index 2 and $X$ is an arbitrary infinite set. Since some nilpotents with index 3 lie in $\mathcal{K}(X)$, we shall determine when $\alpha \in \mathcal{P}(X)$ equals a product of nilpotents with index at most 3. In order to do this, it will be important to know that $d(\lambda)=m$ for any nilpotent $\lambda$. We therefore begin by giving a proof of this fact that is simpler than the one in [9, Lemma 13]. 
LEMMA 3.2. If $m$ is singular and $\lambda$ is a nilpotent then $d(\lambda)=m$ and hence, any product of nilpotents has defect $m$.

PRoof: If $r(\lambda)<m$ then $d(\lambda)=m$. So, we assume $r(\lambda)=m$. Then, by the first paragraph in the proof of [9, Lemma 13], either $g(\lambda)=m$ or $\lambda$ is spread over $m$. If the former occurs, we follow the third paragraph in the proof of $[9$, Lemma 11] (with $\mathrm{cf}(m)$ replaced by $m$ throughout) to conclude that $d(\lambda)=m$. Hence, we assume $\lambda$ is spread over $m$ and suppose, for contradiction, that $d(\lambda)<m$. Let $X \lambda=\left\{x_{i}\right\}$ and $A_{i}=x_{i} \lambda^{-1}$. Since $\aleph_{0}$ is regular, we therefore know there exists $A_{0}$ with $\left|A_{0}\right|>\max \left(\aleph_{0}, d(\lambda)\right)$. Write $A_{0}=B$ and $|B|=n>\aleph_{0}$. If $|X \lambda \cap B|<n$ then $n=|(X \backslash X \lambda) \cap B| \leqslant d(\lambda)$, a contradiction. Hence, $|X \lambda \cap B|=n$ and $B \lambda \neq \emptyset$. Let $J=\left\{i \in I: x_{i} \in B\right\}$, so $|J|=n$. Choose $a_{j} \in A_{j}$ and suppose $\left|X \lambda \cap\left\{a_{j}\right\}\right|<n$. Then $n=\left|(X \backslash X \alpha) \cap\left\{a_{j}\right\}\right| \leqslant d(\lambda)$, a contradiction as before. So $\left|X \lambda \cap\left\{a_{j}\right\}\right|=n$ and $\left\{a_{j}\right\} \lambda^{2} \neq \emptyset$. Repeating the argument, we let $K=\left\{i \in I: x_{i} \in\left\{a_{j}\right\}\right\}$, so $|K|=n$. If $a_{k} \in A_{k}$ then $\left|X \lambda \cap\left\{a_{k}\right\}\right|<n$ provides a contradiction, so $\left|X \lambda \cap\left\{a_{k}\right\}\right|=n$ and $\left\{a_{j}\right\} \lambda^{3} \neq \emptyset$. Clearly, this cannot stop: that is, $\lambda^{r} \neq \emptyset$ for all $r \geqslant 1$, contradicting the fact that $\lambda$ is nilpotent. Hence, $d(\lambda)=m$ as required.

We can now turn to the proof of the following result.

TheOREM 3.3. Suppose $m$ is singular and $\alpha \in \mathcal{P}(X)$. Then $\alpha$ is a product of nilpotents with index at most 3 if and only if $g(\alpha) \geqslant 1, d(\alpha)=m$ and

(a) $g(\alpha) \geqslant r(\alpha)$, or

(b) $\left|z \alpha^{-1}\right| \geqslant r(\alpha)$ for some $z \in X$, or

(c) $g(\alpha) \geqslant \operatorname{cf}(m)$ and $\alpha$ is spread over its rank.

Moreover, when one of (a) - (c) occurs, $\alpha$ can be written as a product of 3 or fewer nilpotents, the first of which may have index 3 and the others have index 2 .

PRoof: If $\lambda$ is a nilpotent and $r(\lambda)<m$ then $d(\lambda)=m$; and if $r(\lambda)=m>\operatorname{cf}(m)$ then $d(\lambda)=m$ by [9, Lemma 13]. Also, if $\lambda$ has index 2 then $X \lambda \subseteq X \backslash \operatorname{dom} \lambda$ implies that (a) is true. Suppose instead that $\lambda$ has index 3 and neither (a) nor (b) hold. Then $r(\lambda)=m$ since $|X| \leqslant r(\lambda)+r(\lambda)^{2}$ by supposition. Hence, by [9, Lemma 13], $\lambda$ is spread over $m$. Let $\operatorname{ker} \lambda=\left\{A_{i}\right\}$ and $J=\left\{i \in I: X \lambda \cap A_{i} \neq \emptyset\right\}$. If $g(\lambda)<\operatorname{cf}(m)$ then $|J|<\operatorname{cf}(m)$ since $X \lambda^{2} \subseteq X \backslash \operatorname{dom} \lambda$ and $|J| \leqslant\left|X \lambda^{2}\right|$. In addition, $|X \lambda \cap \operatorname{dom} \lambda|=m$ : that is, $\left|\bigcup\left(X \lambda \cap A_{j}\right)\right|=m$ where $|J|<\operatorname{cf}(m)$. It follows that not every $X \lambda \cap A_{j}$ can have cardinal less than $m$ (otherwise we invalidate a property of cf $(m)$ : see Theorem 2.1) and so some $z \lambda^{-1}$ has cardinal $m$, contradicting our original supposition. Therefore, $g(\lambda) \geqslant \operatorname{cf}(m)$ and part (c) holds. That is, nilpotents with index 2 or 3 satisfy the specified conditions. Now suppose $\alpha$ is a product of such nilpotents and write $\alpha=\lambda \beta$ where $\lambda$ is one of them. If $g(\alpha)<r(\alpha)$ then $g(\lambda)<r(\lambda)$, so $\lambda$ must satisfy (b) or (c). Suppose some $\left|z \lambda^{-1}\right| \geqslant r(\lambda) \geqslant r(\alpha)$. If $z \notin \operatorname{dom} \beta$ 
then $r(\alpha) \leqslant\left|z \lambda^{-1}\right| \leqslant g(\alpha)<r(\alpha)$ is a contradiction. So, $z \in \operatorname{dom} \beta$ and then $\left|(z \beta) \alpha^{-1}\right| \geqslant r(\alpha)$. On the other hand, if $g(\lambda) \geqslant \operatorname{cf}(m)$ then $r(\alpha)>g(\alpha) \geqslant \operatorname{cf}(m)$ and so, by $[9$, Theorem 4$], \alpha$ is spread over its rank.

By Theorem 2.3, the converse certainly holds whenever (a) holds. For the other two possibilites, let $X \alpha=\left\{x_{i}\right\}$ and $A_{i}=x_{i} \alpha^{-1}$. If some $\left|A_{0}\right| \geqslant r(\alpha)$, write $J=I \backslash 0$ and consider two sub-cases. If $\left|A_{0} \cap X \backslash X \alpha\right|=m$, choose distinct $y_{j}, z_{j}, c \in A_{0} \backslash X \alpha$ as well as $b \notin \operatorname{dom} \alpha$ and note that

$$
\alpha=\left(\begin{array}{cc}
A_{j} & A_{0} \\
y_{j} & b
\end{array}\right) \circ\left(\begin{array}{ll}
y_{j} & b \\
z_{j} & c
\end{array}\right) \circ\left(\begin{array}{cc}
z_{j} & c \\
x_{j} & x_{0}
\end{array}\right)
$$

where the first transformation on the right is nilpotent with index 3 and the other two are nilpotent with index 2. On the other hand, if $\left|A_{0} \cap X \backslash X \alpha\right|<m$ then $\left|\left(X \backslash A_{0}\right) \cap(X \backslash X \alpha)\right|=m$, so we can choose $z_{j}$ and $c$, each different from $b$, inside $\left(X \backslash A_{0}\right) \cap(X \backslash X \alpha)$. Then, if $y_{j} \in A_{0}$, the above decomposition of $\alpha$ will have the same features as before.

Now suppose (c) holds. Since $m$ is singular, it is the sum of $\mathrm{cf}(m)$ cardinals $k_{p}<m$ and, for each $p$, some $A_{p}$ has cardinal greater than $k_{p}$. That is, $\| A_{p} \mid=m$ and we again consider two sub-cases. Put $Q=I \backslash P$. If $\left|\left(\bigcup A_{p}\right) \cap(X \backslash X \alpha)\right|=m$, choose distinct $y_{q}, z_{q}, z_{p} \in\left(\bigcup A_{p}\right) \cap(X \backslash X \alpha)$ as well as $y_{p} \notin \operatorname{dom} \alpha$. Then

$$
\alpha=\left(\begin{array}{ll}
A_{q} & A_{p} \\
y_{q} & y_{p}
\end{array}\right) \circ\left(\begin{array}{ll}
y_{q} & y_{p} \\
z_{q} & z_{p}
\end{array}\right) \circ\left(\begin{array}{ll}
z_{q} & z_{p} \\
x_{q} & x_{p}
\end{array}\right)
$$

where the first trasnformation on the right is nilpotent with index 3 and the other two are nilpotent with index 2 . If instead $\left|\left(\bigcup A_{p}\right) \cap(X \backslash X \alpha)\right|<m$ then $\left|\left(\bigcup A_{p}\right) \cap X \alpha\right|=$ $m$. So, we can choose $y_{q} \in\left(\bigcup A_{p}\right) \cap X \alpha$ and $z_{q}, z_{p} \in(X \backslash X \alpha) \backslash\left\{y_{p}\right\}$ to ensure that the above decomposition of $\alpha$ remains valid.

To show the product 3 is best possible in the above result, we consider the transformation $\alpha$ defined in (1). By Theorem 3.3 (b), $\alpha$ certainly belongs $\mathcal{O}(X)$, the subsemigroup of $\mathcal{L}(X)$ generated by all nilpotents with index at most 3 . Suppose $\alpha=\lambda \mu$ where $\lambda, \mu$ are nilpotents with index at most 3 . If $\lambda$ has index 2 then $r(\lambda) \leqslant g(\lambda)$ and so $m=r(\alpha) \leqslant g(\alpha)=1$, a contradiction. Hence, $\lambda$ has index $3, X \backslash \operatorname{dom} \lambda=\{x\}$, and $\lambda$ acts on $\left\{a_{i}\right\}$ in a one-to-one fashion. Let $a_{i} \lambda=c_{i},\left\{b_{i}\right\} \lambda=\left\{c_{j}\right\}$ and $c_{j} \lambda^{-1}=A_{j}$. Then $x \notin\left\{c_{j}\right\}$ since $\left\{c_{j}\right\} \mu=x$ and $x \mu \neq x$ ( $\mu$ is nilpotent). Hence, $A_{j} \lambda^{2} \neq \emptyset$ but $\left(A_{j} \lambda^{2}\right) \lambda=\emptyset$ : that is, $x=c_{1}$ for some index $1 \in I$ and $c_{j}=a_{1}$ for each $j \in J$. Consequently, $|J|=1$ and $c_{k} \neq x$ for all $k \in K=I \backslash 1$. If $c_{k} \in\left\{a_{k}\right\} \cup\left\{b_{i}\right\}$ then $a_{k} \lambda^{3}=c_{k} \lambda^{2} \in\left(\left\{c_{k}\right\} \cup a_{1}\right) \lambda \neq \emptyset$ is a contradiction. Thus, $c_{k}=a_{1}$ for all $k$ and this contradiction finally proves that $\alpha$ cannot be written as a product of just two nilpotents with index at most 3 . 
Before leaving this section, we show that there are $\alpha \in \mathcal{L}(X)$ which do not satisfy the conditions of Theorem 3.3. For this, choose $Y=\left\{x_{q}\right\} \subseteq X$ with $|Q|=\operatorname{cf}(m)$ as well as some $z \in X \backslash Y$. Then we can write $X \backslash(Y \cup z)=B \cup C$ where $|B|=|C|=m$. Since $m$ is singular, there is a a partition $\left\{A_{q}\right\}$ of $C$ where each $\left|A_{q}\right|<m$. Finally, let $\left\{A_{p}\right\}$ be a partition for $B$, choose $x_{p} \in C$ and put

$$
\alpha=\left(\begin{array}{lll}
A_{p} & A_{q} & Y \\
x_{p} & x_{q} & z
\end{array}\right)
$$

Clearly this is a nilpotent with index 4 that does not satisfy the conditions of Theorem 3.3 ; so, the index 4 in Theorem 3.1 is best possible.

\section{NilPotents as PRODUCTS OF IDEMPOTENTS}

We now turn to the question of whether $\mathcal{L}(X)$ is idempotent-generated. In [8, Section 4], the authors characterised when $\alpha \in \mathcal{P}(X)$ is a product of idempotents in $\mathcal{P}(X)$ by extending the notion of collapse and shift as follows.

$$
\begin{array}{ll}
C^{*}(\alpha)=C(\alpha) \cup X \backslash \operatorname{dom} \alpha & c^{*}(\alpha)=\left|C^{*}(\alpha)\right| \\
S^{*}(\alpha)=S(\alpha) \cup X \backslash \operatorname{dom} \alpha & s^{*}(\alpha)=\left|S^{*}(\alpha)\right|
\end{array}
$$

If $m$ is regular and $\alpha \in \mathcal{L}(X)$ then $d(\alpha)=m$ and either $g(\alpha)=m$ or some $z \alpha^{-1}$ has cardinal $m$ : that is, $c^{*}(\alpha)=m$ and it follows that $s^{*}(\alpha)=m$. Hence, by $[8$, Theorem 8], every element of $\mathcal{L}(X)$ is a product of idempotents in $\mathcal{P}(X)$ : the problem is whether these idempotents can be chosen from $\mathcal{L}(X)$ itself. Note, for example, that if $X=\left\{a_{i}\right\} \cup\left\{b_{i}\right\}$ and

$$
\delta=\left(\begin{array}{c}
\left\{a_{i}, b_{i}\right\} \\
a_{i}
\end{array}\right)
$$

then $\delta$ is an idempotent which lies outside $\mathcal{L}(X)$. As a first step in answering this problem, we now determine when nilpotents in $\mathcal{L}(X)$ with index 2 can be written as a product of idempotents in $\mathcal{L}(X)$.

At the end of [7, Section 2], the authors noted that $K_{m}(Y)$ forms a semigroup for any infinite cardinal $m=|Y|$. And, with this generality in [7, Proposition 3.4], they characterised when a nilpotent with index 2 in $K_{m}(Y)$ is a product of two idempotents in $K_{m}(Y)$. With this in mind, let $\omega$ be the composition of the isomorphism $\theta: \mathcal{L}(X) \rightarrow$ $L_{\phi}$ and the natural map $L_{\phi} \rightarrow L_{\phi} \backslash I_{\phi}=K_{m}(\phi)$ defined in Section 2. If $\alpha^{2}=\emptyset$ in $\mathcal{L}(X)$ and $\alpha=\varepsilon_{1} \varepsilon_{2}$ for some idempotents $\varepsilon_{1}, \varepsilon_{2}$ in $\mathcal{L}(X)$ then $\alpha \omega$ is a product of two idempotents in $K_{m}\left(X^{\phi}\right)$. In addition, if $r(\alpha)=m$ then $\alpha \omega$ is a nilpotent with index 2 in $K_{m}\left(X^{\phi}\right)$. Consequently, by [7, Proposition 3.4], $|C(\alpha \omega) \backslash X(\alpha \omega)|=m$. But, since $g(\alpha) \geqslant 1$, we always have $C(\alpha \omega)=C^{*}(\alpha) \cup \phi$ and clearly $X(\alpha \omega)=X \alpha \cup \phi$. Hence, 
$\left|C^{*}(\alpha) \backslash X \alpha\right|=m$ when $r(\alpha)=m$. On the other hand, if $r(\alpha)<m$ then $d(\alpha)=m$ and so $s^{*}(\alpha)=m$. Therefore, by [8, Theorem 8], $c^{*}(\alpha)=m$ since $\alpha$ is a product of idempotents in $\mathcal{P}(X)$, and so $\left|C^{*}(\alpha) \backslash X \alpha\right|=m$ since $r(\alpha)<m$. That is, we have proved half the following result.

THEOREM 4.1. Suppose $m$ is an arbitrary infinite cardinal and $\alpha \in \mathcal{P}(X)$ is nilpotent with index 2 . Then $\alpha$ is a product of two idempotents in $\mathcal{K}(X)$ if and only if $\left|C^{*}(\alpha) \backslash X \alpha\right|=m$.

Proof: It remains to assume the condition holds and deduce that $\alpha$ is a product ot two idempotents. To do this, write

$$
\alpha=\left(\begin{array}{ll}
B_{t} & u_{q} \\
x_{t} & v_{q}
\end{array}\right)
$$

where each $B_{t}$ contains at least two elements and $\left\{x_{t}\right\} \cup\left\{v_{q}\right\} \subseteq X \backslash$ dom $\alpha$. Choose $b_{t} \in B_{t}$ and put

$$
\begin{aligned}
\varepsilon_{1} & =\left(\begin{array}{ll}
B_{t} & u_{q} \\
b_{t} & u_{q}
\end{array}\right) \\
\varepsilon_{2} & =\left(\begin{array}{cc}
\left\{b_{t}, x_{t}\right\} & \left\{u_{q}, v_{q}\right\} \\
x_{t} & v_{q}
\end{array}\right) .
\end{aligned}
$$

Then $\operatorname{ker} \varepsilon_{1}=\operatorname{ker} \alpha$. Also, $d\left(\varepsilon_{1}\right)=m=g\left(\varepsilon_{2}\right)$ if $|T|=m$ since then $\bigcup\left(B_{t} \backslash b_{t}\right)$ has cardinal $m$ and is contained in $X \backslash X \varepsilon_{1}$ as well as $X \backslash \operatorname{dom} \varepsilon_{2}$. On the other hand, if $|T|<m$ then $\left(C^{*}(\alpha) \backslash X \alpha\right) \backslash\left\{b_{t}\right\}$ has cardinal $m$ and is contained in the same two sets. Also, $d\left(\varepsilon_{2}\right)=d(\alpha)=m$. That is, $\varepsilon_{1}, \varepsilon_{2} \in \mathcal{K}(X)$ and clearly, $\alpha=\varepsilon_{1} \varepsilon_{2}$.

Note that if $X=\left\{a_{i}\right\} \cup\left\{b_{i}\right\}$ and $\alpha$ is the transformation with $\operatorname{dom} \alpha=\left\{a_{i}\right\}$ such that $a_{i} \alpha=b_{i}$ then $\alpha$ is a nilpotent with index 2 which does not satisfy the condition of Theorem 4.1. Despite this, $\alpha$ is a product of idempotents in $\mathcal{K}(X)$. For, suppose $\alpha$ is any nilpotent with index 2 and rank $m$, and write $X \alpha=\left\{x_{i}\right\}$ and $A_{i}=x_{i} \alpha^{-1}$. Choose $a_{i} \in A_{i}$, and write $\left\{a_{i}\right\}=\left\{b_{i}\right\} \cup\left\{c_{i}\right\} \cup y$ and $X \backslash \operatorname{dom} \alpha=\left\{d_{i}\right\} \cup\left\{e_{i}\right\} \cup z$ (possible since $X \alpha \subseteq X \backslash \operatorname{dom} \alpha$ ). Then

$$
\alpha=\left(\begin{array}{cc}
A_{i} & e_{i} \\
d_{i} & z
\end{array}\right) \circ\left(\begin{array}{ll}
d_{i} & e_{i} \\
b_{i} & z
\end{array}\right) \circ\left(\begin{array}{ll}
b_{i} & c_{i} \\
x_{i} & y
\end{array}\right)
$$

where each transformation on the right is a nilpotent with index 2 that satisfies the condition of Theorem 4.1. This leads us to the following result.

THEOREM 4.2. If $m$ is an arbitrary infinite cardinal then $\mathcal{K}(X)$ is idempotentgenerated.

ProOF: Suppose $\alpha$ is nilpotent with index 2. If $r(\alpha)<m$ then $|(\operatorname{dom} \alpha) \backslash C(\alpha)|<$ $m$, so $c^{*}(\alpha)=m$ since $X=(\operatorname{dom} \alpha) \backslash C(\alpha) \cup C^{*}(\alpha)$. Hence, $\left|C^{*}(\alpha) \backslash X \alpha\right|=m$. Therefore, by Theorem 4.1, $\alpha$ is a product of idempotents in $\mathcal{K}(X)$ and, by the above remark, 
the same conclusion holds if $r(\alpha)=m$. Hence, every element of $\mathcal{K}(X)$ is a product of idempotents in $\mathcal{K}(X)$.

We now proceed to show that $\mathcal{L}(X)$ is also idempotent-generated. To do this, we recall that the proof of Theorem 2.2 (see [9, Theorem 3] shows that when $m$ is regular any $\alpha \in \mathcal{L}(X)$ can be written as a product of nilpotents, one of which may have index 3 and take the form:

$$
\lambda=\left(\begin{array}{ll}
A_{i} & Y \\
c_{i} & x
\end{array}\right)
$$

where $\left\{c_{i}\right\} \subseteq Y$ and $x \notin \operatorname{dom} \lambda$. Since the other nilpotents in the product have index 2, by Theorem 4.2 it will suffice to prove that the above $\lambda$ is a product of idempotents in $\mathcal{L}(X)$. So, choose $a_{i} \in A_{i}$ and fix an index $0 \in I$. If $r(\lambda)<m$ then $\left|C^{*}(\lambda) \backslash X \lambda\right|=m$, so we can choose $d_{i} \in\left(C^{*}(\lambda) \backslash X \lambda\right) \backslash\left(\left\{a_{i}\right\} \cup\left\{c_{0}, x\right\}\right)$ since this set has cardinal $m$. Now, observe that

$$
\lambda=\left(\begin{array}{cc}
A_{i} & Y \\
a_{i} & c_{0}
\end{array}\right) \circ\left(\begin{array}{cc}
\left\{a_{i}, d_{i}\right\} & \left\{c_{0}, x\right\} \\
d_{i} & x
\end{array}\right) \circ\left(\begin{array}{cc}
\left\{d_{i}, c_{i}\right\} & x \\
c_{i} & x
\end{array}\right) .
$$

where the first transformation on the right has non-zero gap and same kernel as $\lambda$, so it belongs to $\mathcal{L}(X)$. In addition, the other two transformations on the right have gap equal to $m$, so they belong to $\mathcal{K}(X)$. On the other hand, if $r(\lambda)=m$, we write $\left\{c_{i}\right\}=\left\{c_{j}\right\} \cup\left\{c_{k}\right\}$ where $|J|=|K|=m$ and note that

$$
\lambda=\left(\begin{array}{cc}
A_{i} & Y \\
a_{i} & c_{0}
\end{array}\right) \circ\left(\begin{array}{cc}
a_{i} & \left\{c_{0}, x\right\} \\
a_{i} & x
\end{array}\right) \circ\left(\begin{array}{ccc}
\left\{a_{j}, c_{j}\right\} & a_{k} & x \\
c_{j} & a_{k} & x
\end{array}\right) \circ\left(\begin{array}{ccc}
c_{j} & \left\{a_{k}, c_{k}\right\} & x \\
c_{j} & c_{k} & x
\end{array}\right)
$$

where, as before, each transformation on the right is idempotent and belongs to $\mathcal{L}(X)$. Note in particular that the above decompositions of $\lambda$ as a product of idempotents are valid for any infinite $m$. That is, we have proved part of the following result.

THEOREM 4.3. If $m$ is an arbitrary infinite cardinal then $\mathcal{L}(X)$ is idempotentgenerated.

Proof: It remains to consider the case when $m$ is singular. In this situation, the proof of Theorem 3.1 (see [9, Theorem 4]) shows that any $\alpha \in \mathcal{L}(X)$ can be written as a product of nilpotents, one of which may have index 4 and take the form of (2). But, with the same notation as in (2), we can choose $a_{p} \in A_{p}, a_{q} \in A_{q}, y \in Y$ and put

$$
\begin{aligned}
\varepsilon_{1} & =\left(\begin{array}{lll}
A_{p} & A_{q} & Y \\
a_{p} & a_{q} & y
\end{array}\right) \\
\varepsilon_{2} & =\left(\begin{array}{lll}
a_{p} & a_{q} & y \\
x_{p} & x_{q} & z
\end{array}\right) .
\end{aligned}
$$


Then $\operatorname{ker} \varepsilon_{1}=\operatorname{ker} \alpha$, and $d\left(\varepsilon_{1}\right)=m=g\left(\varepsilon_{2}\right)$ since $\left(\bigcup A_{q}\right) \backslash\left\{a_{q}\right\}$ has cardinal $m$. In addition, $d\left(\varepsilon_{2}\right)=d(\alpha)=m$ by Lemma 3.2. That is, $\varepsilon_{1}$ is an idempotent in $\mathcal{L}(X)$ and, since $\varepsilon_{2} \in \mathcal{K}(X)$ by Theorem 2.3 , it is a product of idempotents in $\mathcal{K}(X)$ by Theorem 4.2. Since the other nilpotents in the decomposition of $\alpha$ have index at most 3 , the result follows from Theorem 4.2 and the above remark.

\section{Further observations}

A slight modification to the proof of Lemma 2.5 shows that $\mathcal{K}(X)$ is regular. For, with the same notation as used there, if $\alpha \in \mathcal{K}(X)$ then $g(\beta)=d(\alpha)=m$ by $[9$, Corollary 3 or Theorem 4]. In addition, $d(\beta)=m$ by the argument in [9, p.341] when $m$ is regular, and by that in the proof of Lemma 2.5 when $m$ is singular. Hence, by [9, Corollary 4], $\beta$ belongs to $\mathcal{K}(X)$. The same argument shows that $\mathcal{O}(X)$ is also regular.

THEOREM 5.1. If $X$ is infinite then $\mathcal{K}(X) \subseteq \mathcal{O}(X) \subseteq \mathcal{L}(X)$ are regular subsemigroups of $\mathcal{P}(X)$, with the second containment being equality when $|X|$ is regular.

As a matter of interest, "we remark that there are transformations with rank less than $m$ at each level in the hierarchy $\mathcal{K}(X) \subseteq \mathcal{O}(X) \subseteq \mathcal{L}(X)$. For, if $m$ is singular, there is a partition $\left\{A_{i}\right\} \cup\{y\}$ of $X$ with $\bigcup A_{i} \mid=m$ but each $\left|A_{i}\right|<m$ and $|I|=$ cf $(m)$. Then, any transformation having $\left\{A_{i}\right\}$ as its kernel must be spread over its rank $\mathrm{cf}(m)$ and have defect $m$, in which case it lies in $\mathcal{L}(X) \backslash \mathcal{O}(X)$. And, when $m$ is regular, it is even easier to find transformations belonging to $\mathcal{O}(X) \backslash \mathcal{K}(X)$. In other words, we cannot write $\mathcal{K}(X)$ or $\mathcal{O}(X)$ as the disjoint union of $I_{m}^{*}$ (see Theorem 2.2) and another subsemigroup of $\mathcal{L}(X)$.

In the previous three sections, we have often used the characterisation of when an element $\alpha$ of $\mathcal{I}(X)$, the symmetric in inverse semigroup on $X$, is a product of nilpotents in $\mathcal{I}(X)$ provided in [9, Corollary 4]: namely, it occurs if and only if $d(\alpha)=g(\alpha)=m$ where $|X|=m$ is an arbitrary infinite cardinal. And, under these conditions, $\alpha$ is a product of 3 or fewer nilpotents in $\mathcal{I}(X)$ with index 2 . An argument identical to that in Theorem 2.7 establishes our next result: it shows that the 3 just mentioned is best possible.

THEOREM 5.2. Suppose $m$ is an arbitrary infinite cardinal and $\alpha \in \mathcal{I}(X)$ is non-zero. Then $\alpha$ is a product of 2 nilpotents in $\mathcal{I}(X)$ with index 2 if and only if $|(X \backslash \operatorname{ran} \alpha) \cap(X \backslash \operatorname{dom} \alpha)| \geqslant r(\alpha)$.

\section{REFERENCES}

[1] A.H. Clifford and G.B. Preston, The algebraic theory of semigroups, Mathematical Surveys 7, vol 1 and 2 (American Mathematical Society, Providence, RI, 1961 and 1967).

[2] J.M. Howie, 'The subsemigroup generated by the idempotents of a full transformation semigroup', J. London Math. Soc. 41 (1966), 707-716. 
[3] J.M. Howie, An introduction to semigroup theory (Academic Press, London, 1976).

[4] J.M. Howie, 'Some subsemigroups of infinite full transformation semigroups', Proc. Roy. Soc. Edinburgh Sect. A 88 (1981), 159-167.

[5] J.M. Howie and M.P.O. Marques-Smith, 'A nilpotent-generated semigroup associated with a semigroup of full transformations', Proc, Roy. Soc. Edinburgh Sect. A 108 (1988), $181-187$.

[6] M.P.O. Marques, 'A congruence-free semigroup associated with an infinite cardinal number', Proc. Roy. Soc. Edinburgh Sect. A 93 (1983), 245-257.

[7] M.P.O. Marques-Smith and R.P. Sullivan, 'Nilpotents and congruences on semigroups of transformations with fixed rank', Proc. Roy. Soc. Edinburgh Sect. A 125 (1995), 399-412.

[8] M.A. Reynolds and R.P. Sullivan, 'The ideal structure of idempotent-generated transformation semigroups', Proc. Edinburgh Math. Soc. 28 (1985), 319-331.

[9] R.P. Sullivan, 'Semigroups generated by nilpotent transformations', J. Algebra 110 (1987), 324-343.

[10] N.H. Williams, Combinatorial set theory, Studies in Logic and the Foundations of Mathematics 91 (North-Holland, Amsterdam, 1977).

Department of Mathematics

University of Western Australia

Nedlands 4009

Australia 